\title{
Discussion and Research of the Concept of Function Teaching under the New Curriculum Standard
}

\author{
Nie Raorong ${ }^{1, a}$ \\ ${ }^{1}$ Nanchang Institute of Science and Technology, Nanchang 330108, China \\ anieraorong@126.com
}

Keywords: New curriculum standards; Function thought; Function teaching

\begin{abstract}
Function occupies very important position in the whole mathematics, and the function is the core concept in the middle school mathematics concept, throughout the entire middle school courses. Function concept itself is of very strong abstractness, which is difficult for students to understand, which cause a lot of problems in the teaching. This article reviewed the function concept and analyzed cognitive level of the concept of function and the influence factors of function concept teaching for high school students. Then put forward some teaching Suggestions under the requirements of new of function concept teaching.
\end{abstract}

\section{The development of function symbols and function concept}

In mathematics, function is the most important and the most basic concept, following the introduction of variable since Descartes, gradually appeared in every field of concepts of variables and functions. Know in the background and development course of function concept, help students understanding the concept of function, and can better understand the function role in development of mathematics.

\subsection{The first expansion of function concept- analytic Functions}

The first expansion of function concept is the British mathematician Greg (J.G regory). In 1667 $\mathrm{AD}$, he in the thesis the theory circle and the curve of the quadrature in the explanation function "from some of the other amount after a series of algebraic operation, or after the operation of other can imagine". But because of the limitations on algebraic operation said the real problem, and imagine operation is a kind of corresponding relation, but Greg was given and the corresponding functions of symbols, so people will soon forget the definition.

John Bernoulli (j. Bernoulli) is the first person to put forward of the concept of function, and in 1694 he was in the study to reflect the movement process and the dependency relationship between variables in the process of movement, his definition is the analytic expression function, namely "variable function is composed of these variables and constants of an analytic expression". [1]And he also uses letters to represent variables $\mathrm{z} \mathrm{n}$ is a function. In 1697, he said about the function method was updated again, use capital letters $X$ or the Greek letter $\xi$ factor variable function. First, in the same period of Jacob Bernoulli also using the letters on the function, he said p and $q$ variable $\mathrm{x}$ any two functions. In 1718 , he created symbol " $\phi x$ ". The word "variable" is also introduced.

\subsection{The second expansion of function concept- function image method}

Well-known German mathematician Leibniz was the first one to use Latin function word "function" as specialized mathematical terms. In a paper, he used the term "function". In 1673 he gave his function definitions, namely "like, of the points on the curve geometry, such as position, string, tangential and normal function is called." At the same time, he stressed that the curve is given in the form of equations. People call the geometric quantity expressed in function as the geometric origins of the concept of "function", this was the beginning of the function of image representation. He also used mark $x, x^{1}, x^{2}$

At the same time, the French mathematician D 'alembert and euler string resonance problems in the research, and must be defined function. D 'alembert's support for its teacher John Bernoulli, in 1698, the given analytical definition, and then euler gave his second function definition: "in the xy 
plane curve of freehand draw indicates the relationship between the $\mathrm{x}$ and $\mathrm{y}$ ".[2]

After function concept is given, it is needed to solve the problem that how symbols corresponding to said that in 1745, D 'alembert proposed his own view, he used $\Delta u, s, \Gamma \mu, s$ for two variables $\mathrm{u}, \mathrm{s}$ function, still use function $\varphi(x)$ to show the funtion of $\mathrm{x}$. In 1753, Euler created $\Phi:(x, t)$ to shoe the function of $x$ and t. In the following yea, he used $f:(a, n)$ to represent the function of $a$ and $n$.

It seems from above that more than three mathematician is represented by the image method function, who nalso had some limitations.

\subsection{The third expansion of function concept-the prototype of the modern concept}

In 1775, Euler conducted expansion in his "differential" on the concept of function for the third time. He gives the definition of the concept of the third function: "if a variable in such a manner depends on other variables, namely when the latter variable changes, the front and the changes of these variables, will be in front of the variables is called the function". Here he still use $f:(x)$ said function. Euler this definition has been called the "rely on" function definitions, it simply reflects the functions are interdependent relationship between variables, and clearly from the "change" to the process of "for" show in front of people, so, it was thought that definition is the prototype of the modern concept of function[3].

The famous French mathematician Lagrange also gave the definition of function in 1797. He actually used the definition of power series functions, and vigorously promoted the use of English mark " $f$ " and " $F$ )" and Greece alphabet " $\Phi " ~ " \Psi "$ function. Under the influence of him, maths still used the $f(x), F(x), \Phi(x), \psi(x)$ to represent function.

\section{Function teaching under the "curriculum standard"}

\subsection{The goal of function teaching requirements in the "curriculum standard"}

We know math function is one very important and plays a role of commander in chief is in the middle school mathematics, LianXiMian also widely important core concept. In the ordinary high school mathematics curriculum standard (experiment) ", the knowledge required for the function, content and approach on the big changes has taken place. Function in the curriculum content to consider the problem is on the basis of inheriting traditional teaching material advantage, demand from the general process of the concept of function, nature and application of the concept of function and use of information technology to help students understand the function concept up to break through the difficulty.

In junior high school students have been learning function based on an example of the concept and some specific functions, such as a function, the inverse function, quadratic function, etc., and in the high school stage is the contents of the further learning function. In the high school stage, the essence of the more prominent function, dependencies, corresponding relation, a special relationship, and symbolic symbols to represent, understanding and the understanding of the essence of the function in the spiral; [4]And increased the function model and its application, because the function is to depict reality change rule (movement) model, so whether functional concepts, or basic elementary function, are as models to study.

"Ordinary high school mathematics curriculum standard" pointed out: in the teaching should be the basic concept and basic thought of intensive, understand and grasp the core concepts and basic thoughts more always in high school mathematics teaching, to help students deepen understanding gradually. In mathematics thinking activity is the concept, judgment and reasoning, while the concept is the basis of judgment and reasoning. Concept is thinking of cells, only in the concept under the premise of clear understanding, to master operation skills and techniques, also will have reasonable logical argumentation.

\subsection{Links between middle school mathematics function teaching with other contents}

Function of knowledge in mathematics and other subjects have a wide range of application: function and equation, the function and the inequality, function with close links to the series and so 
on; Movement and the unity of opposites is the concept of function view embodied in mathematics; Function concept and reflect the mathematical thought has already permeated various fields of mathematics; Function is the foundation of learning higher mathematics knowledge. Therefore, research on the subject to high school students learning and teachers' teaching has certain reference value.

\subsubsection{The relation and distinction between function and equation}

Function and equation includes three meanings: function thought, equation of thought as well as the function and equation into each other's minds. Function is a pointer to the real problem of material, on the wide range of association and imagination, through the establishment of function model, applying the concept of function properly and nature to analyze problem, transformation and solve problems; Equation thought from the problem, by analyzing the quantity relationship by using mathematical language conditions of the problem can be converted to equation or equations model, and then equation or equations to get answer problem. In order to make the problems solved better, function thoughts and equation thought most can be mutual conversion.

\subsubsection{The relationship and difference between function and inequality}

In many cases, the function and the inequality is also can be mutual conversion between. Inequality is actually a function in the function values greater than zero or less than zero, if the images of its reflected in rectangular coordinate system, the function of the corresponding is the entire curve, and inequality corresponding is just located above or below the $\mathrm{x}$ axis, the quadratic function, for example, its expression is $\left.y=a x^{2}+b x+c\right)(a \neq 0)$, when $y>0$ or $y<0$, it is converted to a yuan quadratic inequality students familiar with. Quadratic function of the image in plane rectangular coordinate system is a parabola, the abscissa parabola and $\mathrm{x}$ axis intersection points between two side and part is corresponding to a yuan quadratic inequalities solution.

\subsubsection{Connections between secondary function and higher mathematics in function content}

German mathematician f. klein's work is the high point of elementary mathematics "(" elementary mathematics" refers to the German elementary and middle schools mathematics, slightly higher than our country middle and primary school mathematics), points out that university and middle school mathematics education there is a "double disconnect" phenomenon. College students may feel that he is learning knowledge and high school studied has nothing to do, and when they went to the school, and university studies on the use of. [5] His purpose of writing this book is to want to change this kind of unreasonable phenomenon, want to make the mathematics education "modernization". He believes that teachers should have a higher mathematical point of view, because the higher the view, the more the simple things. Such as in the real domain to understand in the complex domain can see; In Euclidean space is bad to explain phenomena, from the point of view of projective space can explain, etc. Klein, points out that in middle school about traditional logarithmic term is a loophole, he suggested that the logarithmic function is introduced as the area under the isometric hyperbola, simple and clear; He pointed out that in the complex domain, can see more clearly the essence of the logarithmic function: this is because the logarithmic function, as the logarithm of real Numbers is just one of countless multiple values.

\section{The influencing factors of function concept teaching}

Based on the analysis of students' questionnaire, it is found that many students know function concept, but can't understand the question, answers to questions was answered incorrect, do not understand $y, f(x)$, several kinds of representation can't apply the function, main findings through questionnaire and social actual situation has the following several ways affect students' understanding of the concepts of function.

\subsection{Social factors}

In middle school mathematics, the concept of function is the most basic concept, is the focus of teaching, but also a difficult point for students to learn. In actual teaching, the college entrance examination pressure, many teachers worry about the problem solving training time is not enough to use, in a hurry end of teaching concept, conclusion, formula, and then exams tactics to improve 
the students' ability of problem solving. Because the student did not really understand and grasp the essence of the concept, will cause the obstacles of students' problem solving problem solving ability of students is hard to improve. In the social environment, some parents still to score the stand or fall of evaluating a school quality of teaching, it is forced to social pressure.

\subsection{The function concept itself}

From the students' answer can be found in the questionnaire are students' understanding of the concepts of function surface, this function factors of the concept itself has a lot to do.

First, the function contains content has the diversity and complexity, we know that the function concept map is the essential attribute and the map to meet two conditions is arbitrary and uniqueness, that is to say nature is arbitrary and uniqueness of the concept of function, the function concept, however, still have a lot of essential attribute, such as domain, domain and so on.

Second, the function definition written expression form is with strong logic.

Third, the function concept is a concept with syndrome differentiation, function of expression is both a variable and constant; Is a practical significance for each individual and the whole of, containing, change and movement of thought.

Fourth, the symbol function $f(x)$ is hard to see from the surface its practical significance. Even if the " $f$ " represented by corresponding laws has been given, the student to the symbol " $f$ " and said the specific content is difficult to set up contact.

\subsection{The student thought development level}

Middle school students' thinking development level of general performance for the specific image thinking gradually transition to formal logic thinking, in the process of thinking formation, the student is not dependent on the perceptual experience and gradually rising slowly from the experience of abstract thinking to the theory of abstract thinking, high school students in the form of logical thinking level have a certain degree of increase, but their dialectical thinking is still in the early formation and development.

Function is characterized by the development and changes, and other concepts closely linked, it is a process of dynamic change, but most of the students' dialectical thinking is not very mature, their thoughts can't between movement and stillness, continuous and discrete flexible transformation, they can only static, separation, local learned, abstract understanding of things. This creates a student is not whole, dynamic understanding function, are not good at the abstract concept of function associated with concrete living examples.

So, if want to make the students have correct understanding of function concept, we have to help them break through the limitations of formal logic thinking.

\section{Summary}

Because the middle school classroom is still guided by the college entrance examination, coupled with the limited class time, and cross of each course, students often rarely think better. Teachers also pay more attention to students' problem solving ability, leaving limited time in guiding students in autonomous learning. The author thinks that, to make students to master relevant knowledge function better, teachers should not only explain to the students question about mathematical concept, but also had better do good thinking before teaching, fully prepared for a variety of strategies and the status of the students in the teaching process more observation. After teaching, teachers should reflect on the summary, and often communicate with his colleagues. It is believed that they will have new insights and harvest.

\section{References}

[1] Chen Bei. Function concept of teaching and learning: [D]. Jiangsu province, Yangzhou university, 2016.

[2] You Xiaoping. Function concept teaching thinking and practice [J]. Journal of middle school mathematics magazine, 2014. 
[3] Li Jibao. Several problems on the concept of function teaching [J]. Journal of mathematics education, 2013.

[4] Zhong Zhimin. High student function of the corresponding relationship to understand [J]. Shanghai: east China normal university, 2013.

[5] Ceng Zheng. On the teaching of mathematics concept and the overall thinking [J]. Journal of zhaoqing university, 2012. 